\title{
The Manganite-Water Interface
}

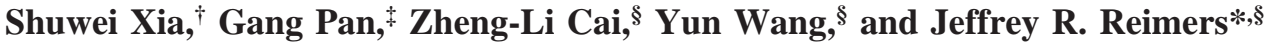 \\ College of Chemistry and Chemical Engineering, Ocean University of China, Qingdao 266003, China, State \\ Key Laboratory of Environmental Aquatic Chemistry, Research Centre for Eco-Environmental Sciences, \\ Chinese Academy of Sciences, Beijing 100085, China, and School of Chemistry, The University of Sydney, \\ NSW 2006, Australia
}

Received: December 21, 2006; In Final Form: May 9, 2007

\begin{abstract}
The properties of bulk manganite, its freshly cleaved (010) surface, and this surface exposed to water monolayers at both low and high coverage and to liquid water at $300 \mathrm{~K}$, are examined using density-functional theory (DFT) by the PW91 density functional as well as using the new PM6 semiempirical electronic-structure method. The bonds between the (010) layers are calculated to be very weak, of average energy $-2.7 \mathrm{kcal}$ $\mathrm{mol}^{-1}$, explaining the ease at which manganite surfaces cleave. Upon cleavage, a surface reconstruction is predicted that produces ferroelectrically ordered surface layers, and the surface manganese atoms are predicted to display different chemical properties depending on the nature of the oxygen atom to which they bind in the subsurface layer. Water is predicted to be only physisorbed to the surface, with this process acting to lift the surface reconstruction. At high water coverage, the differences between the two types of surface manganese atoms are also lost. Simulations at $300 \mathrm{~K}$ indicate that less than half of the surface manganese atoms coordinate to the fluid at $300 \mathrm{~K}$ while only two-thirds of the manganite oxygen atoms on the outside of the surface coordinate. No dominate liquid structure is found, suggesting that dielectric continuum models may be useful in understanding surface chemistry, but it is clear that water-water rather than water-surface interactions dominate the nature of the interface.
\end{abstract}

\section{Introduction}

Manganese is an abundant element in the earth's crust, existing mainly as oxides and hydroxides found often at the interfaces between the lithosphere and either the hydrosphere, atmosphere, or biosphere. ${ }^{1}$ In particular, manganese nodules cover $10-30 \%$ of the floor of the Pacific Ocean ${ }^{2}$ and are very common in sediment-water interfaces; their propensity to undergo both redox and acid/base reactions thus leads to these materials providing a major controlling factor for groundwater chemistry. ${ }^{2}$ Manganese oxide chemistry is also commercially important through its use as a hydrogen-storage medium in alkaline dry-cell batteries. ${ }^{3}$ While much is now known concerning the detailed bulk structure of the some 30 manganese oxides/ hydroxides, far less information is available concerning the nature of the corresponding surfaces. Manganite, or $\gamma-\mathrm{MnOOH}$, is known to be important in regulating the concentration, reactivity, and toxicity of many pollutants such as lead, zinc, and cadmium in the aquatic environment. These interactions can now be measured at the atomic/molecular level using synchrotron based technologies such as XAFS (X-ray Adsorption Fine Structure).$^{4-9}$ However, it is very difficult to apply these spectroscopic methods to complex systems, especially when adsorbate concentrations are low. There is thus considerable interest in the development of a fundamental understanding of manganite's pre-eminent ${ }^{10}(010)$ surface and its chemistry in aqueous solution. In this work we study the interaction between this surface and water by means of a priori electronic structure and molecular dynamics calculations.

\footnotetext{
* Corresponding author. E-mail: reimers@chem.usyd.edu.au.

Ocean University of China.

$\div$ Chinese Academy of Sciences.

$\S$ The University of Sydney.
}

The complete bulk structure of $\gamma-\mathrm{MnOOH}$ was determined by Dachs ${ }^{11}$ in 1963 using combined neutron and X-ray diffraction techniques, and this has been recently improved by Kohler et al. ${ }^{12}$ using high-resolution X-ray diffraction. The original crystal structure reported an orthorhombic cell containing eight equivalent $\mathrm{MnOOH}$ units per cell, this designation arising due to twinning associated with the locations of the hydrogen atoms, while the more recent study finds a smaller monoclinic unit cell comprising just four $\mathrm{MnOOH}$ units with long-range proton disorder. Both descriptions depict the same atomic arrangements and hydrogen-bonding topology. Shown in Figure 1 are eight copies of the orthorhombic cell in a $2 \times 2 \times 2$ configuration, displaying 256 atoms. The oxygen atoms in each $\mathrm{MnOOH}$ unit are distinct and are conventionally named $\mathrm{O} 1$ and $\mathrm{O} 2$, while the proton sits at a location between them forming a covalent bond to $\mathrm{O} 1$ and a hydrogen bond to $\mathrm{O} 2$. The manganese atoms are octahedrally coordinated by three $\mathrm{O} 1$ atoms and three $\mathrm{O} 2$ atoms, arranged such that all linear bond angles are of the form $\mathrm{O} 1-\mathrm{Mn}-\mathrm{O} 2$. Each oxygen atom has tetrahedral coordination, covalently bonded to three manganese atoms and either covalently bonded or hydrogen bonded to a hydrogen atom. As the $\mathrm{O} 1-\mathrm{O} 2$ separation is very small, $2.6 \AA$, the hydrogen bonding is very strong and the $\mathrm{O} 1-\mathrm{H}-\mathrm{O} 2$ link should be thought of in general terms as a three-center four-electron system; full treatment of the quantized motion of the hydrogen atom in this environment is required for the calculation of its properties. To highlight both this feature and the overall topology, all $\mathrm{O} 1-\mathrm{H}-\mathrm{O} 2$ links shown in Figure 1 and subsequent figures are presented with both linkages marked equivalently.

It is clear from Figure 1 that manganite forms a layered structure in the (010) direction, with each manganese atom 


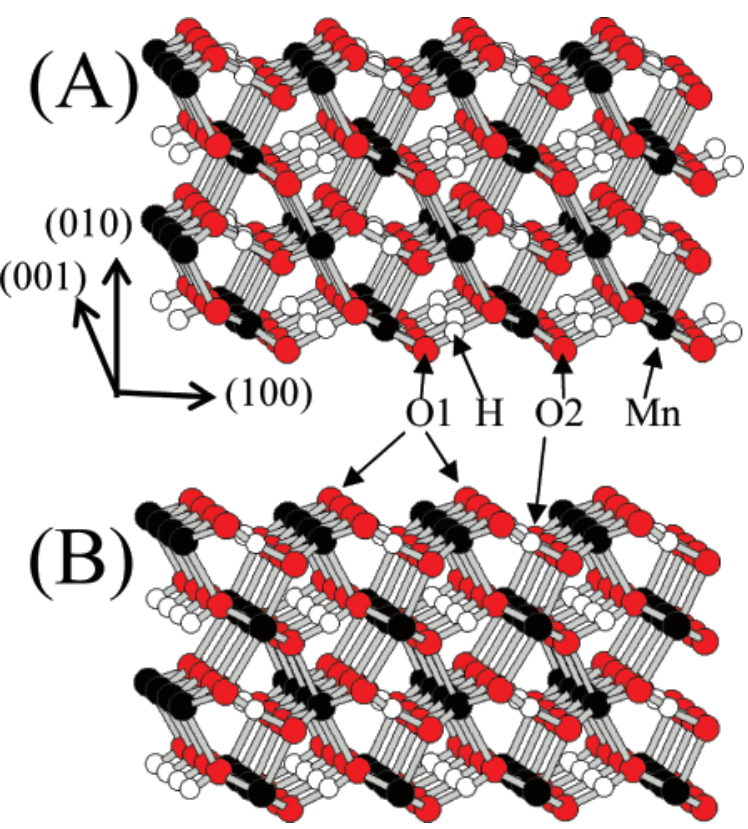

Figure 1. PW91-optimized structures (soft pseudopotential) for bulk manganite, showing the directions of the lattice vectors and the distinct types of manganese, oxygen, and hydrogen atoms. Shown are eight images of the orthorhombic unit cell, each image showing eight $\mathrm{MnOOH}$ units, with the $(010)$ cleavage planes oriented in the vertical direction. The $\mathrm{O} 1-\mathrm{H}$ (covalent) and $\mathrm{O} 2-\mathrm{H}$ (hydrogen) bonds are equivalently depicted to emphasize the nature and topology of the $\mathrm{O} 1-$ $\mathrm{H}-\mathrm{O} 2$ linkages. The structure in panel $\mathrm{A}$ is antiferroelectric within each layer, while that in panel B comprises alternatively aligned ferroelectric layers.

forming four bonds within each layer and one bond to each of the neighboring layers. Further, Jahn-Teller distortion lengthens and weakens the interlayer bonds considerably, and each oxygen also forms three (covalent or hydrogen) bonds within a layer and one interlayer (covalent) bond. Hence, cleavage along (010) planes dominates manganite surface production. ${ }^{10}$ Per orthorhombic unit cell, the freshly cleaved (010) surface thus features eight broken bonds, four involving manganese atoms and four involving oxygen atoms, and these sites are susceptible to chemical attack. Indeed, the surface is known to be subject to chemisorption reactions involving the solvent. At $\mathrm{pH} 6$, the surface is protonated at a density of $0.55 \mu \mathrm{mol} \mathrm{m} \mathrm{m}^{-2}$ or 1 proton per 7 surface cells, while at $\mathrm{pH} 10$ the surface is negatively charged with one charge per 2.5 cells $;{ }^{4}$ the isoelectronic point is at $\mathrm{pH}$ 8.2. However, both the nature of the uncharged surface in terms of the degree of chemisorption or physisorption of the solvent, and the details of the ensuing acid/base reactions, remains to be determined.

The (010) surface is also subject to oxidative corrosion, ${ }^{2,4}$ and hence, it is possible that freshly cleaved surfaces rapidly develop irregularities making step edges, corners, etc., the dominating features of the surface chemistry. Indeed, apparent surface coverages of up to two zinc or cadmium atoms per reactive site have been observed, ${ }^{6,7}$ suggesting that the actual surface area is greater than that expected for a flat surface. However, while low-resolution atomic-force microscopy (AFM) images of the surface ${ }^{13}$ show evidence of some pitting, there does not appear to be large-scale pore formation. It thus appears that the properties of the flat newly cleaved surface may be relevant, making this topology a natural starting point for the investigation of manganite surface chemistry.

Bulk manganite has previously ${ }^{3}$ been modeled a priori using density-functional-theory (DFT) simulations employing the Perdew-Wang 1991 (PW91) density functional, ${ }^{14}$ a functional derived using a generalized-gradient approximation. It was demonstrated to predict values of the cell parameters and heavyatom bond lengths that are very close to the experimentally observed ones, and the many investigated properties have been found to be well reproduced. ${ }^{3}$ However, the $\mathrm{O} 1-\mathrm{H}$ bond length is predicted to be $1.114 \AA$, much longer than that of $0.98 \pm$ $0.02 \AA$ from the latest X-ray structural refinement ${ }^{12}$ but close to the value of $1.019 \AA$ obtained in 1963 using both X-ray and neutron diffraction techniques. ${ }^{11}$ The experimental value is in good agreement with that predicted from correlations relating observed $\mathrm{OH}$ bond lengths, vibration frequencies, and oscillator strengths, ${ }^{15,16}$ indicating inadequacies in the previous calculations. Clearly, however, PW91 has been shown to provide an excellent description for most of the properties of manganite and related materials, ${ }^{3}$ and it is also known to provide a generally reliable description of liquid water. ${ }^{17}$

Unfortunately, DFT approaches are computationally expensive as well as being subject to systematic failures ${ }^{18}$ (though no such failure is expected for manganite). A new semiempirical method, Parametrized Model 6 (PM6) introduced by Stewart ${ }^{19}$ is currently in its beta development phase. It is the first method of this type parametrized for (at least) every element of the periodic table excepting the lanthanides and actinides, and it offers significant enhancements in computational efficiency compared to PW91, a highly desired feature for the simulation of chemical processes at solid-liquid interfaces and for materials science research in general. Methods such as PM6 also suffer from systematic failures owing to their empirical treatment of electron correlation, but more significantly each element must be individually parametrized and so the performance of the method must also be determined on an element-by-element basis. While PM6 has been demonstrated to provide excellent results for organic molecules and many properties of more general systems, detailed applications to the chemistry and solidstate physics of manganese are yet to be developed. Such studies are required in order to optimize the parameters so that PM6 will be able to describe manganese in a wide range of chemical environments.

In this work we simulate the structure of the freshly cleaved, uncharged, regular, manganite (010) surface, considering initially the induced changes to the proton structure using the PW91 and PM6 methods. The previously reported ${ }^{3}$ inadequacies in $^{2}$ the $\mathrm{O} 1-\mathrm{H}-\mathrm{O} 2$ bond lengths are alleviated and the quantized vibrational levels are computed for both bulk and surface protons. The binding of water molecules with the surface to form monolayers at both low and high coverage is then investigated, considering the possibility of both physisorbed and chemisorbed layers. Structures are evaluated at temperatures of $0 \mathrm{~K}$, by geometry optimization, and at $300 \mathrm{~K}$, by DFT molecular dynamics (DFT-MD). Finally, structures are obtained using DFT-MD for samples of liquid water sandwiched between two manganite (010) surfaces.

\section{Methods}

All DFT calculations are performed using the VASP computer package $^{20,21}$ using ultrasoft pseudopotentials to describe the effective core potential of the atoms. ${ }^{22,23}$ Two pseudopotentials of this type are available for oxygen, and both are used herein, a soft one that is computationally efficient and a hard one that provides an improved description of $\mathrm{O}-\mathrm{H}$ interactions. A planewave basis set is used truncated using energy cutoffs of 300 and $500 \mathrm{eV}$ for the soft and hard pseudopotentials, respectively; these are set slightly in excess of the minimum recommended values of 270 and $396 \mathrm{eV}$ to improve convergence. 
Calculations for bulk manganite are performed using the smallest-possible orthorhombic ${ }^{11}$ unit cell, a cell that contains eight $\mathrm{MnOOH}$ units, double that of the smallest-possible monoclinic ${ }^{12}$ unit cell. Most simulations involving manganite surfaces are performed taking two copies of this cell replicated in the (010) direction, inserting a vacuum region of at least 10 $\AA$ extent so as to produce a three-dimensional periodic system comprised of alternating slab and vacuum regions. This is described as being a $1 \times 1$ (in the (100) and (001) directions) surface cell, and is four manganite layers (4L) thick; some calculations are also performed using a slab that is six layers (6L) thick. As manganite is nonmetallic and all of the unit cells used are of moderate to large size, all calculations are performed at the gamma point of the Brillouin zone.

In surface hydration studies, either $1,8,18$, or 28 water molecules are inserted into the vacuum region between the manganite slabs; the eight-molecule samples represent highcoverage water monolayers on the surface while the larger samples represent liquid water sandwiched between the slabs. For the liquid samples, the box length in the (010) direction is optimized to reduce to zero the pressure for a singly selected configuration. Hence, while these simulations are performed at constant volume and are not sufficiently extensive to calculate the associate pressure, the simulation conditions do provide a realistic approximation to ambient conditions. In another liquid simulation, a $1 \times 2$ surface unit cell is chosen containing 56 water molecules; the initial structure for this simulation prior to thermalization was obtained by replicating a structure from the $1 \times 128$-molecule simulation. All manganite slabs contain four (010) layers of which the central two are frozen at the PW91-optimized coordinates of bulk manganite; only the surface layers are allowed to vary during the simulations.

The initial structure of liquid water sandwiched between the manganite surfaces was obtained by molecular dynamics using the MM+ force field in HYPERCHEM, ${ }^{24}$ run at $300 \mathrm{~K}$ for 5 ps at a frozen manganite structure. The resulting configuration was then quenched to $0 \mathrm{~K}$ and used as the starting configuration for the PW91 calculations. These commenced by thermalizing the structures for $0.5-1.0 \mathrm{ps}$ and the structural properties were then averaged for $1-5 \mathrm{ps}$. All molecular visualization was also performed using HYPERCHEM. ${ }^{24}$ All DFT-MD calculations are performed using the "VeryFast" computational algorithm with a time step of $1 \mathrm{fs}$ and kinetic energy renormalization (so as to emulate a canonical ensemble at finite temperature) every $20 \mathrm{fs}$; the initial velocity vectors are selected from a random canonical distribution and are typically reset after every $500 \mathrm{fs}$ of simulation.

In manganite, the manganese atoms have a $\mathrm{d}_{4}$ high-spin configuration. Calculations were performed based on spinrestricted Kohn-Sham orbitals (low spin) as well as open-shell ferromagnetic and antiferromagnetic high-spin states. Evaluated using the soft pseudopotential at the geometry of the observed structure $^{11}$ of manganite, the energy of the low-spin state fell by $39 \mathrm{kcal} \mathrm{mol}^{-1}$ (of manganese atoms) on formation of the ferromagnetic state and then again by $4.0 \mathrm{kcal} \mathrm{mol}^{-1}$ on formation of the lowest-energy antiferromagnetic state. Manganite is indeed known to be antiferromagnetic. ${ }^{2,3}$ A variety of antiferromagnetic states are possible depending on the alignment chosen for the spins in neighboring atoms, and all possibilities were investigated and the lowest-energy state found. This state has within each (010) layer alternating atomic spins for manganese atoms in the first and second coordination shells around a manganese atom; the next-lowest energy configuration was well separated at an excess energy of $3 \mathrm{kcal} \mathrm{mol}^{-1}$.
All PM6 calculations were performed using the beta-release version of MOPAC 7.2 that also embodies the beta-release version of the PM6 parameters. ${ }^{19}$ Antiferromagnetic states were produced by first obtaining the density for the corresponding ferromagnetic state, manually interchanging the alpha and beta electron densities according to the pattern previously determined using PW91, and restarting the PM6 calculation specifying a singlet spin-unrestricted wave function. This procedure is analogous to that commonly used to study diradical states, including for example the properties of $\mathrm{H}_{2}$ at bond lengths beyond the singlet - triplet instability radius. ${ }^{25}$ All calculations were performed on unit cells containing two replicas of the basic orthorhombic cell in the (001) direction, while in addition two replicas in the (010) direction were also used for bulk manganite. Such large cells were generated party due to the requirement introduced by the method used to treat the Brillouin-zone integration in MOPAC that all cell vectors must exceed $7 \AA$ in length. However, the expansion in the (001) direction also provides for significant enhancement in the description of liquid water above the surface.

\section{Results and Discussion}

a. Optimized Structures for the Bulk Solid and the Freshly Cleaved Slabs. The PW91-optimized structure obtained using the soft pseudopotential for bulk manganite is shown in Figure 1 and closely parallels that which has been previously reported; ${ }^{3}$ small differences arise as these previous calculations considered the smaller monoclinic unit cell using an extensive $4 \times 4 \times 4$ $k$-point mesh and a ferromagnetic arrangement of the electrons. Using the hard pseudopotential produces no qualitative changes to this structure, but the quantitative differences are reported in Table 1 along with the corresponding properties obtained using PM6. Also, the analogously calculated properties for the bare (010) surface are also listed in this table, while full coordinates for all structures are provided in Supporting Information.

For the bulk solid, the PW91-calculated Mn-O distances, both for the short bonds within each layer and the long bonds between layers, are within $0.03 \AA$ of the latest experimental results, ${ }^{12}$ with the errors being slightly larger when the hard pseudopotential for oxygen is used. The cell parameters are at variance by up to $0.10 \AA$, the errors again being larger for the harder pseudopotential; the calculated total cell volume of 271.3 $\AA^{3}$ for the soft pseudopotential is in excellent agreement with the observed ${ }^{12}$ value of $270.4 \AA{ }^{3}$ while that for the hard pseudopotential is $3 \%$ too large at $278.6 \AA .{ }^{3}$ A significant feature is that the PW91 calculations qualitatively predict the observed antiferromagnetic electronic structure and antiferroelectric proton structure, see Methods section. However, as previously noted for manganite, ${ }^{3}$ the soft pseudopotential predicts the covalently bonded $\mathrm{O} 1-\mathrm{H}$ distances to be too long by the significant amount of $0.09 \pm 0.02 \AA$, and the corresponding O1-O2 separation of $2.50 \AA$ is $0.10 \AA$ shorter than the experimental value. PW91 typically underestimate ${ }^{26,27}$ hydrogen-bonded $\mathrm{O}-\mathrm{O}$ separations by $0.1 \AA$ but calculated $\mathrm{OH}$ bond lengths are usually overestimated $^{27,28}$ by only ca. 0.01 . Use of the less-approximate hard pseudopotential significantly removes this anomaly, predicting $\mathrm{OH}$ bond lengths in excess of experiment by just $0.01 \AA$ and O-O separations to within $0.02 \AA$. PW91 is in general known to provide a realistic description of the energetics of hydrogenbond formation. ${ }^{26,29-31}$

The calculated energy for the cleavage of the bulk lattice along a (010) vector of the four-layer cell is calculated by PW91 to be 31.8 (41.3) $\mathrm{kcal} \mathrm{mol}^{-1}$ per surface cell using the hard(soft) pseudopotential. As this process involves cleavage of four 
TABLE 1: Observed and Calculated (by PM6 or PW91 Using Hard and Soft Pseudopotentials) Lattice Parameters $a, b, c$ (in $\AA ̊$ ), Cell Volume (in $\AA^{3}$ ) and Internuclear Distances (in $\AA$ ) for Manganite Bulk Solid and for the Atoms in Slabs Exposing Its (010) Freshly Cleaved Surface ${ }^{a}$

\begin{tabular}{|c|c|c|c|c|c|c|c|c|c|c|c|c|c|c|}
\hline sample & method & size & struct. $^{b}$ & $a$ & $b$ & $c$ & vol. & $\mathrm{Mn}-\mathrm{O} 2 \mathrm{~L}$ & $\mathrm{Mn}-\mathrm{O} 1 \mathrm{~L}$ & $\mathrm{Mn}-\mathrm{O} 2$ & $\mathrm{Mn}-\mathrm{O} 1$ & $\mathrm{O} 1-\mathrm{H}$ & $\mathrm{O} 2-\mathrm{H}$ & $\mathrm{O} 2-\mathrm{O} 1$ \\
\hline \multirow[t]{7}{*}{ bulk } & obs. ${ }^{c}$ & $1 \times 1 \times 1$ & $\mathrm{aFE}$ & 8.88 & 5.25 & 5.71 & 266.2 & 2.20 & 2.33 & 1.88 & 1.97 & 1.019 & 1.565 & 2.58 \\
\hline & obs. $^{d}$ & $1 \times 1 \times 1$ & $\mathrm{aFE}$ & 8.915 & 5.277 & 5.748 & 270.4 & 2.21 & 2.34 & $1.88,1.89$ & $1.98,1.98$ & $0.98 \pm 0.02$ & 1.590 & 2.60 \\
\hline & PW91/soft & $1 \times 1 \times 1$ & $\mathrm{aFE}$ & 8.840 & 5.293 & 5.800 & 271.3 & 2.24 & 2.35 & $1.90,1.91$ & $1.97,1.97$ & 1.073 & 1.426 & 2.50 \\
\hline & PW91/soft & $1 \times 1 \times 1$ & FE & [8.840] & [5.293] & [5.800] & [271.3] & 2.23 & 2.36 & 1.92 & 1.96 & 1.084 & 1.402 & 2.49 \\
\hline & PW91/hard & $1 \times 1 \times 1$ & $\mathrm{aFE}$ & 8.917 & 5.348 & 5.843 & 278.6 & 2.25 & 2.39 & $1.91,1.89$ & $2.00,1.99$ & 1.028 & 1.552 & 2.58 \\
\hline & PW91/hard & $1 \times 1 \times 1$ & $\mathrm{FE}$ & 8.890 & 5.386 & 5.885 & 284.6 & 2.24 & 2.43 & 1.92 & 2.00 & 1.035 & 1.546 & 2.58 \\
\hline & PM6 & $1 \times 2 \times 2$ & $\mathrm{FE}$ & 9.272 & 12.079 & $\infty$ & $\infty$ & 2.08 & $\infty$ & 1.88 & 2.02 & 1.011 & 1.698 & 2.71 \\
\hline \multirow[t]{7}{*}{ (010) slab } & PW91/soft & $1 \times 14 \mathrm{~L}$ & $\mathrm{FE}$ & [8.840] & {$[21.66]^{e}$} & [5.800] & & 2.21 & 2.26 & 1.91 & 1.92 & 1.054 & 1.491 & 2.53 \\
\hline & PW91/soft & $1 \times 14 \mathrm{~L}$ & $\mathrm{AFE}^{f}$ & [8.840] & {$[21.66]^{e}$} & [5.800] & & 2.19 & 2.24 & $1.91,1.97$ & $1.93,1.97$ & $1.053,1.089$ & $1.380,1.488$ & $2.47,2.5$ \\
\hline & PW91/hard & $1 \times 14 \mathrm{~L}$ & $\mathrm{FE}$ & [8.917] & {$[21.66]^{e}$} & [5.843] & & 2.18 & 2.24 & 1.91 & 1.93 & 1.018 & 1.613 & 2.62 \\
\hline & PW91/hard & $1 \times 14 \mathrm{~L}$ & $\mathrm{aFE}$ & [8.917] & {$[21.66]^{e}$} & [5.843] & & 2.21 & 2.28 & $1.86,1.90$ & $1.95,2.00$ & 1.046 & 1.512 & 2.56 \\
\hline & PW91/hard & $1 \times 16 \mathrm{~L}$ & $\mathrm{FE}$ & [8.917] & {$[32.09]^{e}$} & [5.843] & & 2.18 & 2.25 & 1.91 & 1.94 & 1.017 & 1.619 & 2.63 \\
\hline & PW91/hard & $1 \times 16 \mathrm{~L}$ & aFE & [8.917] & {$[32.09]^{e}$} & [5.843] & & 2.21 & 2.27 & $1.86,1.90$ & $1.95,1.99$ & 1.055 & 1.462 & 2.52 \\
\hline & PM6 & $1 \times 24 \mathrm{~L}$ & $\mathrm{FE}$ & [8.840] & {$[21.66]^{e}$} & [11.60] & & 2.29 & 2.34 & 1.89 & 1.99 & 1.068 & 1.528 & 2.59 \\
\hline
\end{tabular}

${ }^{a}$ Atoms are labeled as per Figure 1, and $L$ indicates distances to a different (010) layer. ${ }^{b}$ Proton structures: aFE, antiferroelectric arrangement within each layer; FE, ferroelectric arrangement of the surface layer of the slab or alternating oppositely polarized ferroelectric layers of bulk manganite; see Figures 1 and 2. ${ }^{c}$ From Dachs, ${ }^{11}$ space group $P 2_{1} / d$ (orthorhombic $\mathrm{Mn}_{8} \mathrm{O}_{16} \mathrm{H}_{8}$ ). ${ }^{d}$ From Kohler et al., ${ }^{12}$ space group $P 2{ }_{1} / d$ (orthorhombic

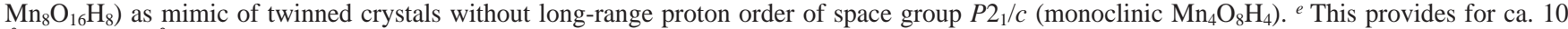
$\AA$ (4-L) or $16 \AA$ (6-L) of vacuum between the periodically replicated manganite slabs. ${ }^{f}$ Optimized with frozen hydrogen locations to inhibit collapse to the FE structure.
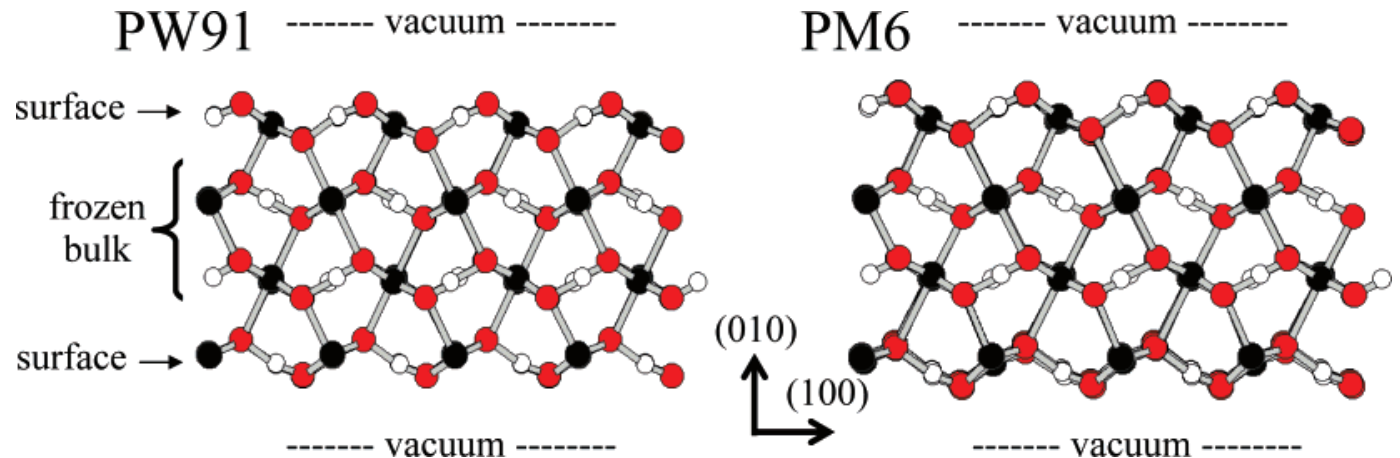

Figure 2. Optimized structures obtained using PW91 (soft pseudopotential) or PM6 for a slab of manganite (black, manganese; red, oxygen; white, hydrogen, with both $\mathrm{O} 1-\mathrm{H}$ covalent bonds and $\mathrm{O} 2-\mathrm{H}$ hydrogen bonds marked equivalently). The inner atomic layers are frozen at the PW91-optimized coordinates of bulk manganite while the surface atoms only are allowed to relax. Two replicas of the superlattice unit cell in the (100) direction are shown for clarity. The surface layers display a ferroelectric arrangement of their protons.

$\mathrm{Mn}-\mathrm{O}$ bonds as well as four $\mathrm{O}-\mathrm{Mn}$ bonds, the average strength of each broken bond is just $4.0(5.2) \mathrm{kcal} \mathrm{mol}^{-1}$. Using the sixlayer cell, this energy drops to just $2.7 \mathrm{kcal} \mathrm{mol}^{-1}$. Hence it is clear that the driving force for the manganese atoms to be 6-coordinate and the oxygen-atoms to be four-coordinate is very small. Indeed, the layers are held together by forces that are only of the strength of weak hydrogen bonds, and this is why manganite crystals tend to flake. ${ }^{10}$

Seemingly quite poor results are predicted using the betarelease parametrization of PM6, however. While the correct antiferromagnetic structure does result, PM6 migrates half of the protons within each layer, effectively interchanging the positions of the $\mathrm{O} 1$ and $\mathrm{O} 2$ atoms as these are defined as the ones that are covalently bonded and hydrogen bonded, respectively, to these protons. This process creates pairs of layer that are internally connected by strong $\mathrm{Mn}-\mathrm{O} 2$ bonds but externally connected to other paired layers through only $\mathrm{Mn}-\mathrm{O} 1$ bonds, bonds that subsequently dissociate and break the crystal apart. Hence PM6 predicts that manganite is not stable. As a result, the applicability of PM6 in its current trial form to study the surface properties of the mineral is questioned. The results do serve to focus attention toward important properties of manganite, however: the weakness of its interlayer bonds, and the additional strength of $\mathrm{Mn}-\mathrm{O} 2$ interactions compared to $\mathrm{Mn}-$ O1 ones.

Both PW91 (using either the hard or soft pseudopotentials) and PM6 predict that four-layer slabs of manganite in vacuum are stable, and the optimized structures, constrained to keep the PW91-optimized values for the in-layer lattice constants $a$ and $c$, are shown in Figure 2. Both structures are similar although the surface layers from PM6 show more distortion than those from PW91. Most significantly, both methods predict that half of the surface protons transfer between their surrounding oxygens, thus effectively interchanging the locations of these surface $\mathrm{O} 1$ and $\mathrm{O} 2$ atoms. As a result, the surfaces become ferroelectric, with all $\mathrm{OH}$ dipoles aligning to give a nonzero component in the (100) direction. In the four-layer cell used, these fluctuations in the two surface layers are anticorrelated so that the whole system has no net dipole moment. The rearrangement places all $\mathrm{O} 1$ atoms at the extremium of the surfaces while all $\mathrm{O} 2$ atoms appear on the inside of the surface layers and form links to the subsurface layers. As a result, the surface $\mathrm{OH}$ groups become all highly exposed to the environment. Note that it is the same chemical processes that cause this reconstruction that lead to the predictions of either very weak (PW91) or nonexistent (PM6) interlayer adhesion in the bulk material.

An alternate structure for the bulk was then considered in which each layer contains a ferroelectric arrangement of the protons, with the dipole vectors of adjacent layers aligning in opposite directions so as to produce a net antiferroelectric lattice. This structure was optimized using PW91 with both the soft and hard pseudopotentials and local minima found. Both structures have the same qualitative appearance and this is shown 


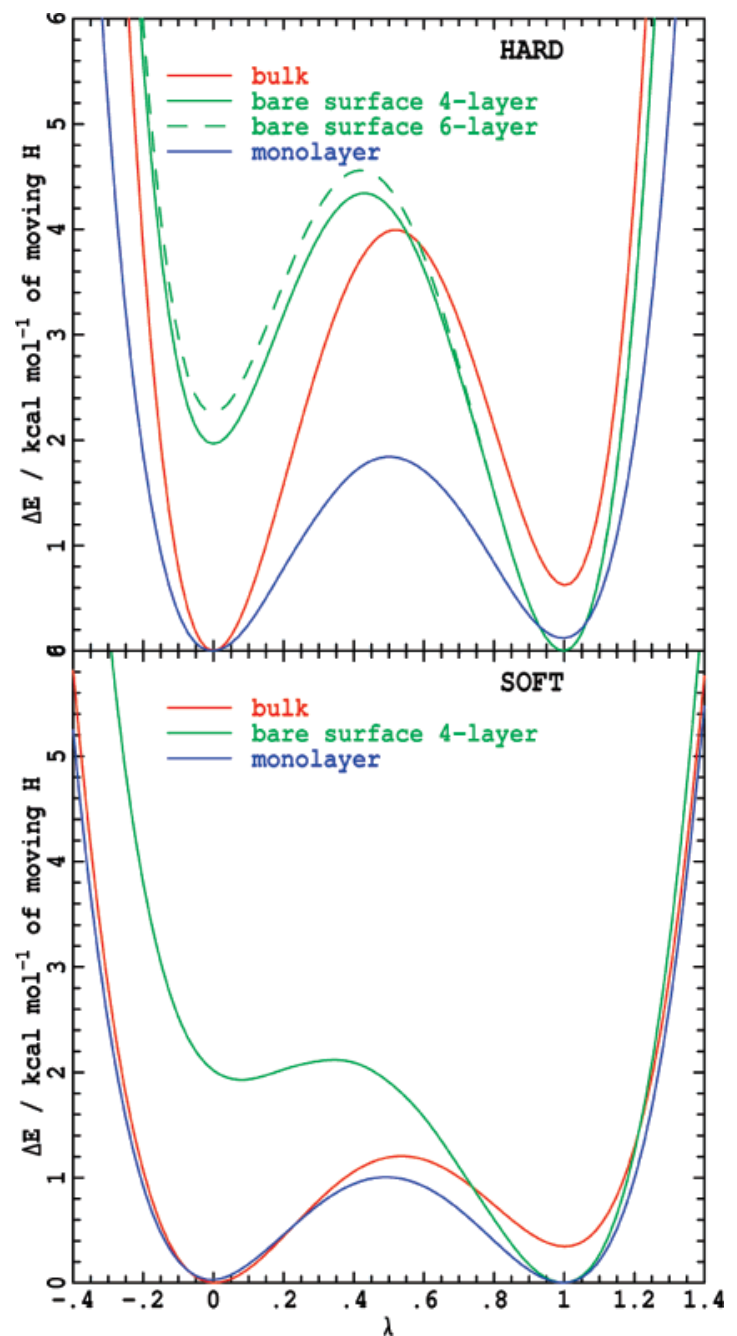

Figure 3. Potential energy changes calculated using either hard (upper) or soft (lower) plane-wave energy cutoffs, $\Delta E$, for proton rearrangements in the bulk solid, on the bare (010) surface (from 4-layer and 6-layer models), and on the (010) surface (4-layer model) covered by a water monolayer, obtained by linear interpolation between the optimized antiferroelectric $(\lambda=0)$ and in-layer ferroelectric $(\lambda=1)$ structures described in Tables 1 and 2.

in Figure 1; key properties are summarized in Table 1 and full details are provided in Supporting Information. For the orthorhombic $1 \times 1 \times 1$ unit cell considered, these structures are higher in energy than the ferroelectric structures by just 2.0 or $0.50 \mathrm{kcal} \mathrm{mol}^{-1}$ per hydrogen atom for this proton-transfer process.

In a parallel result, using the hard pseudopotentials that provide enhanced description of $\mathrm{O}-\mathrm{H}$ interactions, a highenergy bulk-like antiferroelectric surface structure was also obtained. For the soft pseudopotential, this structure is unstable but a representative model was obtained by constraining the hydrogen-atom locations; this antiferroelectric structure is shown in Figure 2. To understand the stability of the ferroelectic and antiferroelectric bulk and surface structures, PW91 potentialenergy surfaces are constructed by linear interpolation, and the results are shown in Figure 3. On this figure, the points at $\lambda=$ 0 are for the optimized antiferroelectric structures, those at $\lambda=$ 1 are for the optimized ferroelectric ones, while intermediary local maxima provide upper bounds to the energies of the associated transition states. This figure shows that while the computationally expedient but less accurate soft pseudopotential provides a very good description of the relative energies, it significantly underestimates the barriers separating them: near

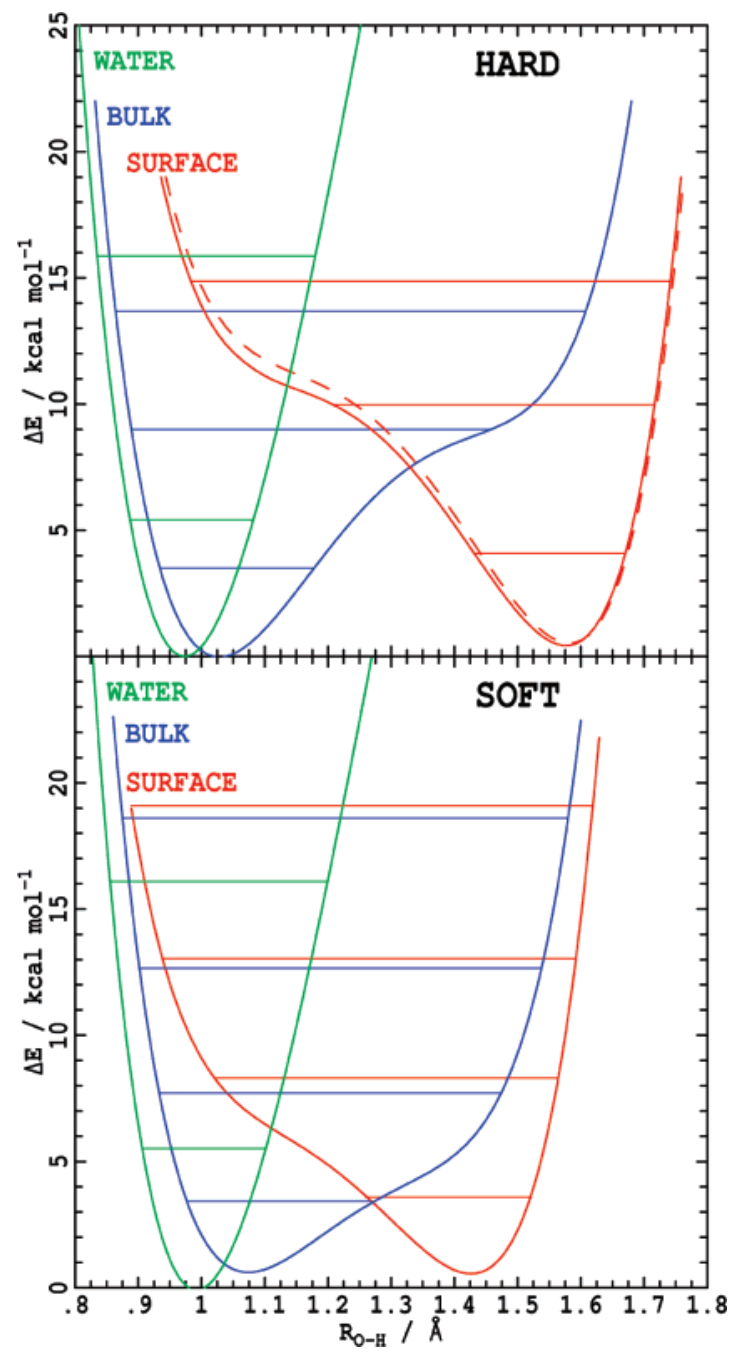

Figure 4. PW91 potential-energy differences calculated using hard (top) and soft (bottom) pseudopotentials from the fully optimized minimum-energy structure (Table 1, Figures 1 and 2), $\Delta E$, obtained by placing one of the $\mathrm{H}$ atoms from the optimized bulk, slab, and water monomer structures at a distance $R_{\mathrm{O}-\mathrm{H}}$ from one of the oxygen atoms along the vector toward the closest oxygen. Analogous oxygen atoms are considered in both the bulk and slab structures so that the surfaces reflect the change from antiferroelectric alignment of the $\mathrm{OH}$ bonds in the bulk to ferroelectric alignment on the surface. The anharmonic vibrational energy levels obtained from these potentials are also shown.

zero instead of $4.3 \mathrm{kcal} \mathrm{mol}^{-1}$ per proton for the surface protons and 1.3 instead of $4.0 \mathrm{kcal} \mathrm{mol}^{-1}$ per proton for the bulk.

A key factor influencing the structural and electronic properties of both the bulk and the surface is the degree of charge polarization (ionicity) of the atoms. Atomic charges have been determined for the lowest-energy bulk and surface structures using PW91 (Bader charges ${ }^{32,33}$ ) for both the hard and soft pseudopotentials and PM6 (Mulliken charges), and the results are given in Table 2 . The manganese atoms, which are formally $\mathrm{Mn}^{3+}$ ions, have charges of 1.6 to 1.8 and 1.2 e from the PW91 and PM6 analyses, respectively, reflecting a significant degree of covalency in the manganese-oxygen bonds. PM6 predicts oxygen and hydrogen charges of ca. -0.7 e $(\mathrm{O} 1),-0.8$ e $(\mathrm{O} 2)$, and $0.35 \mathrm{e}(\mathrm{H})$, typical of what is found for these atoms in water and in the hydrophilic parts of proteins; it thus perceives manganite as having a network molecular structure. However, PW91 predicts that manganite is much more like an ionic solid, with atomic charges of -1.2 to $-1.3 \mathrm{e}(\mathrm{O} 1),-1.1 \mathrm{e}$ to $-1.2 \mathrm{e}$ $(\mathrm{O} 2)$, and $0.7 \mathrm{e}(\mathrm{H})$, with in particular strong charge polarization involving the bonded $\mathrm{O} 1$ and $\mathrm{H}$ atoms. Both PW91 and PM6 
TABLE 2: PW91 and PM6 Calculated Atomic Charges (in Units of e) for Atoms in Bulk Manganite and in Manganite Slabs of Various Layer Thicknesses Obtained Using Both Soft and Hard Pseudopotentials in PW91 Calculations

\begin{tabular}{|c|c|c|c|c|c|c|c|c|c|c|c|c|c|c|}
\hline \multirow[b]{2}{*}{ sample } & \multirow[b]{2}{*}{ layers } & \multirow[b]{2}{*}{ atoms } & \multicolumn{4}{|c|}{ PW91/hard } & \multicolumn{4}{|c|}{ PW91/soft } & \multicolumn{4}{|c|}{ PM6 } \\
\hline & & & $\mathrm{Mn}$ & O1 & $\mathrm{O} 2$ & $\mathrm{H}$ & $\mathrm{Mn}$ & O1 & $\mathrm{O} 2$ & $\mathrm{H}$ & $\mathrm{Mn}$ & O1 & $\mathrm{O} 2$ & $\mathrm{H}$ \\
\hline bulk & & all & 1.58 & -1.24 & -1.05 & 0.71 & 1.83 & -1.30 & -1.22 & 0.69 & $1.23,1.12$ & -0.71 & -0.77 & 0.34 \\
\hline slab & 4 & center & 1.58 & -1.21 & -1.05 & 0.69 & 1.84 & -1.36 & -1.20 & 0.71 & $1.26,1.13$ & -0.70 & -0.80 & 0.36 \\
\hline slab & 4 & surface & 1.55 & -1.17 & -1.07 & 0.68 & 1.81 & -1.26 & -1.20 & 0.66 & 1.06 & -0.71 & -0.77 & 0.34 \\
\hline slab & 6 & center & 1.58 & -1.25 & -1.04 & 0.71 & & & & & & & & \\
\hline slab & 6 & surface & 1.55 & -1.17 & -1.07 & 0.68 & & & & & & & & \\
\hline
\end{tabular}

indicate that the electronic structure of the surface is very similar to that of the bulk, providing another indication of the weakness of the interlayer interactions as well as an indication that adsorbate interactions with the surface will be weak. This notwithstanding, both methods also indicate that the surface is hydrophilic. Note that the PW91 results are reasonable insensitive to the hardness of the pseudopotential used, as well as to the number of layers used in the slab calculations.

b. Isolated $\mathbf{O 1}-\mathbf{H}-\mathbf{O 2}$ Vibrational Motions. The potentialenergy surfaces shown in Figure 3 depict the energy per moving proton of lattice modes that change the nature of the crystal structure. These are not the energy surfaces felt by individual protons, however, as in principle an infinite number of protons must move in order to sustain this motion. In practice, such phonon modes will be short ranged, however, resulting perhaps in grain boundaries between regions of different proton registration. The simplest such scenario is the motion of a single proton in its local environment without correlated motions of adjacent protons. To model this process, Figure 4 shows the onedimensional PW91 potential energy surface obtained by placing one of the hydrogen atoms of the bulk crystal at a location along the $\mathrm{O} 1-\mathrm{O} 2$ vector and then optimizing all atoms except this one hydrogen. It is placed at a distance $R_{\mathrm{OH}}$ away from the atom that is of type $\mathrm{O} 1$ in the bulk. The corresponding potentialenergy surfaces for longer-wavelength correlated $\mathrm{OH}$ motions can be obtained through the appropriate combinations of the potential-energy surfaces from Figures 3 and 4. For transfer of a single proton, the double-minimum type potential energy surface often associated with proton-transfer reactions is not obtained as the proton-transferred structure includes manganese atoms linked now to either two or four O1 atoms instead of the usual three, making this structure apparent in Figure 4 as a shoulder at ca. $4 \mathrm{kcal} \mathrm{mol}^{-1}$ (soft pseudopotential) or $8 \mathrm{kcal}$ $\mathrm{mol}^{-1}$ (hard pseudopotential) of excess energy. Allowing the oxygen atoms to relax during this process lowers the energy by a small amount but does not change the qualitative nature of the proton-transfer process. Significant differences are seen between the results obtained using soft and hard pseudopotentials, with the underestimated $\mathrm{OH}$ bond lengths obtained using the soft pseudopotentials resulting in a broader vibrational well. Shown also in Figure 4 are energy surfaces for gas-phase water monomer obtained by stretching one its local $\mathrm{OH}$ oscillators away from equilibrium. Comparison of this surface to those obtained for manganite indicates the profound effect that the hydrogen bonding has on width of the $\mathrm{OH}$ potential-energy well.

The quantized energy levels obtained for the frozen-environment $\mathrm{OH}$ potential-energy surface of the bulk are indicated on Figure 4. For water monomer, the calculated harmonic vibration frequency is 3834 (3899) $\mathrm{cm}^{-1}$ for the hard (soft) pseudopotential, reducing to $3703(3655) \mathrm{cm}^{-1}$ after anharmonic correction. This later result is close to the observed average $\mathrm{OH}$ water vibration frequency of $3745 \mathrm{~cm}^{-1}$, indicating that the PW91 method realistically treats $\mathrm{OH}$ vibrations. For bulk manganite, the calculated harmonic vibration frequencies is 2654 (2187) $\mathrm{cm}^{-1}$, reducing to 1919 (1460) $\mathrm{cm}^{-1}$ after anharmonic correc- tion. The frequency lowering arises from the dramatic increase in the width of the well apparent in Figure 4, but its value significantly exceeds the observed frequency lowering ${ }^{12,15}$ which is to just $2627 \mathrm{~cm}^{-1}$. The calculated values are, however, in good agreement with the observed correlation between expectation values of $\mathrm{OH}$ bond lengths and $\mathrm{OH}$ vibration frequencies, ${ }^{16}$ while the observed value is consistent ${ }^{16}$ with the observed bondlength expectation value of $0.98 \AA$, as is the observed oscillator strength. ${ }^{15}$ It is hence clear that the slightly overestimated $\mathrm{OH}$ bond length of water obtained using the hard pseudopotential of $0.974 \AA$ compared to $0.9572 \AA$ observed contributes to the error in the calculated frequency. However, it is clear that PW91 underestimates the energy of proton transfer, an effect possibly associated with its large perceived ionicity of the crystal, as discussed earlier.

Also shown in Figure 4 is the analogous potential-energy surface for the motion of a hydrogen atom on the surface of the manganite slab. The selected atom is one for which the equilibrium position changes on cleavage of the surface. Table 1 shows that the calculated covalent equilibrium bond length decreases from 1.028 (1.073) $\AA$ in the bulk to 1.017 (1.046) $\AA$ on the surface using the hard(soft) pseudopotential, and Figure 4 shows that this occurs because the relative energy of the hydrogen-transferred structure is increased compared to the bulk due to the preference for O2-type atoms to bind with the subsurface layer. As a result, the calculated $\mathrm{OH}$ anharmonic vibration frequency is predicted to increase by $141(150) \mathrm{cm}^{-1}$ to 1919 (1610) $\mathrm{cm}^{-1}$ on surface cleavage. This frequency increase is expected to be a robust effect arising from the desire of the inner-surface $\mathrm{O} 2$ atoms not to interact with their external environment.

c. The Possibility of Water Chemisorption on the Manganite (010) Surface. In aqueous solution, the manganite surface is known to be quite sensitive to $\mathrm{pH}$, displaying amphoteric properties, ${ }^{4}$ and the raw cleaved surface contains manganese atoms with missing bonds to oxygen as well as oxygen atoms with missing bonds to manganese. These properties allow for the possibility that water dissociatively chemisorbs to the manganite surface, with $\mathrm{OH}^{-}$terminating the broken bonds to surface manganese and $\mathrm{H}^{+}$terminating broken bonds to surface oxygens. Such a structure would be stabilized by internal hydrogen bonds between the water fragments, as well as by interactions with the liquid water. While a thorough analysis of the energetics of this process requires extensive free-energy calculations, the primary driving force for chemisorption would be the strength of the surface interactions. This has been investigated by placing $\mathrm{OH}$ and $\mathrm{H}$ groups at the vacant bulklattice sites above the surface, optimizing the structure using PW91 with the soft pseudopotential. Both surfaces of the manganite slab were included in these calculations. PW91 predicted that chemisorbed structures are unstable, however, preferring physisorbed water attached to manganese. As there are two types of manganese atoms on the surface (those bonded to $\mathrm{O} 1$ or $\mathrm{O} 2$ atoms in the subsurface layer), and at least two types of surface oxygen, possible scenarios also exist in which 


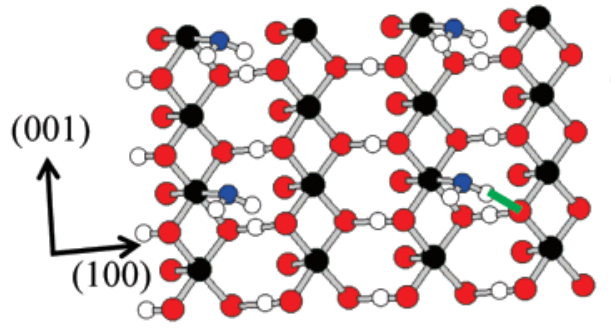

Low Coverage (soft)
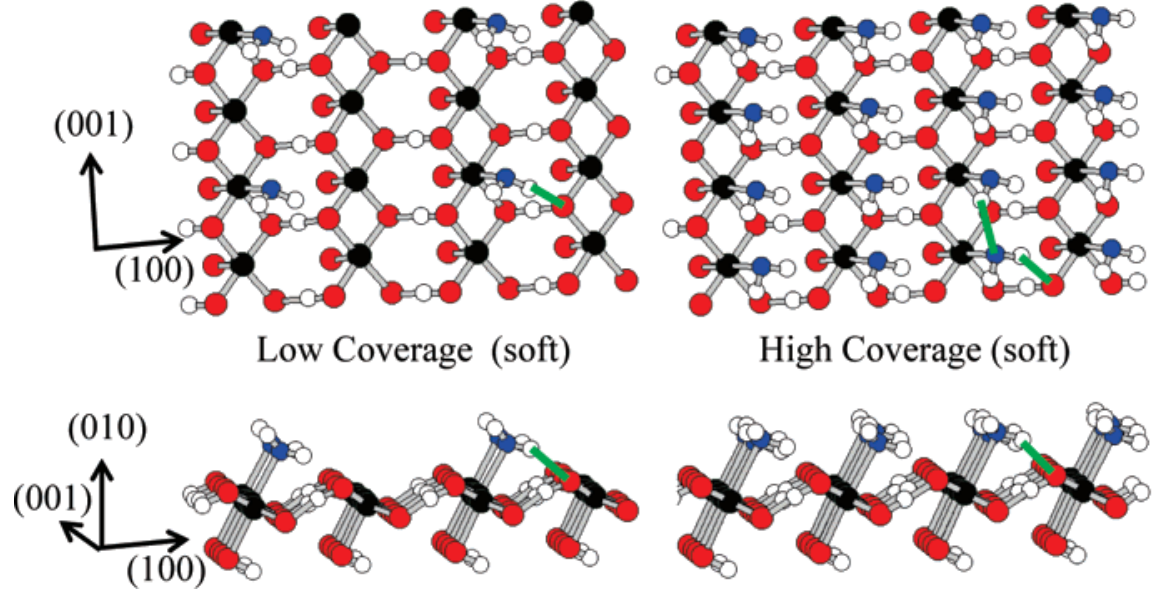

High Coverage (soft)

Figure 5. PW91 soft-pseudopotential optimized structures $\mathbf{1}$ and $\mathbf{4}$ at low and high coverage, respectively, and the hard-pseudopotential optimized structure 6a at high coverage, for water physisorbed to a manganite (010) surface. For clarity, only a single manganite layer is shown while two replicas of the optimized unit cell in each of the (100) and (001) directions are provided. Black, manganese; white, hydrogen; red, manganite oxygen; blue, water oxygen; green, sample hydrogen bonds involving the water adsorbate.

TABLE 3: PW91 Calculated Properties Using Soft and Hard Pseudopotentials (PP) for Water Molecules Physisorbed to Manganite (010) at Low or High Coverage (One or Four Water Molecules Per Four Surface Manganese Atoms, Respectively) ${ }^{a}$

\begin{tabular}{|c|c|c|c|c|c|c|c|c|c|c|c|c|c|c|c|c|c|c|c|c|}
\hline \multirow[b]{2}{*}{ \# } & \multirow[b]{2}{*}{ PP. } & \multirow[b]{2}{*}{ cov. } & \multirow[b]{2}{*}{$\begin{array}{c}\text { surf. } \\
\text { OH } \\
\text { struct. }^{c}\end{array}$} & \multirow[b]{2}{*}{$\begin{array}{l}\text { Water } \\
\text { orient. }^{d}\end{array}$} & \multicolumn{4}{|c|}{$\Delta E$} & \multicolumn{6}{|c|}{$R$ for network involving $\mathrm{Mn} 1^{b}$} & \multicolumn{6}{|c|}{$R$ for network involving $\mathrm{Mn} 2^{b}$} \\
\hline & & & & & total & mang. & water & int. & $\begin{array}{c}\mathrm{OO}- \\
\mathrm{H}\end{array}$ & $\begin{array}{l}\mathrm{Mn}- \\
\mathrm{OW}\end{array}$ & $\begin{array}{c}\mathrm{OO}- \\
\mathrm{OW}\end{array}$ & $\begin{array}{l}\mathrm{OO}- \\
\mathrm{HW}\end{array}$ & OWOW & $\begin{array}{c}\mathrm{HW}- \\
\mathrm{OW}\end{array}$ & $\begin{array}{c}\mathrm{OO}- \\
\mathrm{H}\end{array}$ & $\begin{array}{c}\mathrm{Mn}- \\
\mathrm{OW}\end{array}$ & $\frac{\mathrm{OO}-}{\mathrm{OW}}$ & $\begin{array}{c}\mathrm{OO}- \\
\mathrm{HW}\end{array}$ & OWOW & $\begin{array}{c}\mathrm{HW}- \\
\mathrm{OW}\end{array}$ \\
\hline 1 & soft & low & $\sim \mathrm{FE}$ & para. & -11.1 & 4.9 & 0.6 & -16.6 & - & - & - & - & - & - & 1.294 & 2.30 & 2.79 & 1.86 & - & - \\
\hline 2 & soft & low & $\mathrm{FE}$ & para. & -8.3 & 5.5 & 0.3 & -14.1 & 1.077 & 2.31 & 2.97 & 2.16 & - & - & - & - & - & - & - & - \\
\hline 3 & soft & high & $\mathrm{aFE}$ & para. & -11.9 & 1.9 & -2.8 & -11.0 & 1.063 & 2.38 & 2.92 & 2.09 & 2.92 & 2.07 & 1.413 & 2.55 & 2.86 & 1.93 & 2.88 & 2.11 \\
\hline 4 & soft & high & $\mathrm{aFE}$ & para. & -11.8 & 1.9 & -2.9 & -10.8 & 1.065 & 2.58 & 2.84 & 1.91 & 2.95 & 2.1 & 1.419 & 2.38 & 2.95 & 2.12 & 2.88 & 2.09 \\
\hline 5 & soft & high & FE & perp. & -6.1 & 0.1 & -4.7 & -1.6 & 1.056 & 4.72 & 3.06 & 2.09 & 3.73 & 3.05 & 1.058 & 3.53 & 2.82 & 1.96 & 2.82 & 1.89 \\
\hline 6 & soft & high & $\mathrm{FE}$ & para. & -11.8 & 1.1 & -3.0 & -9.9 & 1.070 & 2.59 & 2.97 & 2.13 & 2.91 & 2.06 & 1.085 & 2.41 & 2.89 & 1.99 & 2.90 & 2.09 \\
\hline $4 a$ & hard & high & $\mathrm{aFE}$ & para. & -10.3 & 1.9 & -3.6 & -8.6 & 1.02 & 2.34 & 2.94 & 2.23 & 2.86 & 1.93 & 1.51 & 3.11 & 3.05 & 2.30 & 3.14 & 2.40 \\
\hline $6 a$ & hard & high & $\mathrm{FE}$ & para. & -10.2 & 0.9 & -3.6 & -7.5 & 1.03 & 2.32 & 3.20 & 2.37 & 2.88 & 1.96 & 1.03 & 3.12 & 3.12 & 2.23 & 3.13 & 2.38 \\
\hline
\end{tabular}

${ }^{a} \Delta E$ is the interaction energy (in $\mathrm{kcal} \mathrm{Mol}^{-1}$ of adsorbate) in total and its contributions from the change in the manganite structure, the water structure and water-water interactions, and the manganite-water interaction. $R$ are the bond lengths (in $\AA$ ) between atoms of type: OO, surface outside oxygen; $\mathrm{H}$, surface hydrogen; Mn, surface manganese; OW, oxygen of water; and HW, hydrogen of water. ${ }^{b} \mathrm{Mn} 1$ and Mn2 are surface manganese atoms bonded to ligands of type $\mathrm{O} 1$ and $\mathrm{O} 2$ in the subsurface layer, respectively. ${ }^{c}$ Surface-layer $\mathrm{OH}$ structures: aFE, antiferroelectric arrangement with OOs alternating between $\mathrm{O} 1$ and $\mathrm{O} 2$; FE, ferroelectric arrangement with all $\mathrm{OO}$ as $\mathrm{O} 1$; $\sim \mathrm{FE}$, all $\mathrm{FE}$ with $\mathrm{OO}$ as $\mathrm{O} 1$ except for the $\mathrm{OO}$ that receives the hydrogen bond which is $\mathrm{O} 2 .{ }^{d}$ Orientation of the planes of the water molecules, largely either parallel (see, e.g., Figure 5) or vertical to the surface.

some water molecules are physisorbed to the surface while others are chemisorbed. All possibilities were investigated and the purely physisorbed structure was preferred in all cases.

d. Water Physisorption on the Manganite (010) Surface at Low and High Coverage. Physisorbed water monolayers have been considered at both low coverage (one water per four surface manganese atoms) and high coverage (one water per surface manganese atom), and a variety of structures were optimized using both the PW91 and PM6 methods. Except for two test structures, only soft pseudopotentials are used, and all of slabs used were four layers thick. Some low-energy configurations obtained through this procedure are shown in Figure 5 while energetic and geometric properties for these are other representative structures are given in Table 3; the optimized coordinates for all of the discussed structures are provided in Supporting Information. As all computational methods yield qualitatively similar results, only those obtained using PW91 with soft pseudopotentials are explicitly shown.

At low-coverage, the water molecules are isolated from each other and interact primarily with the manganite surface. The water molecules coordinate to surface manganese atoms, additionally offering hydrogen bonds to surface oxygens. On the surface there are two types of manganese atoms, however, to which the calculated binding strengths are significantly different. These are manganese atoms bonded to $\mathrm{O} 1$ or $\mathrm{O} 2$ atoms in the subsurface layer, named $\mathrm{Mn} 1$ and $\mathrm{Mn}$ 2, respectively; the interaction energy (Table 3 ) is $-11.1 \mathrm{kcal} \mathrm{mol}^{-1}$ coordinated to $\mathrm{Mn} 2$ and $-8.3 \mathrm{kcal} \mathrm{mol}^{-1}$ coordinated to Mn1. The surface $\mathrm{OH}$ structure retains the ferroelectric nature of the raw cleaved surface when the water coordinates to $\mathrm{Mn} 1$ but the stronger interaction with $\mathrm{Mn} 2$ returns the associated surface proton to its bulk-like antiferroelectric structure. It is hence clear that the manganite surface is easily modified by its environment.

The bulk-based classification of oxygen atoms as either $\mathrm{O} 1$ or $\mathrm{O} 2$ depending on its bonding does not provide a useful description of these processes as the surface is composed of oxygen atoms in significantly different environments, those on the outside that are directly exposed to the solvent, and those on the inside that ligate to the subsurface layer. We hence introduce a new classification of the surface oxygens as either OO (oxygen on the outside) or OI (oxygen on the inside). In addition, the water hydrogen and oxygen atoms are named HW and $\mathrm{OW}$, respectively. Using this notation, the key structural changes induced by the hydrogen bonding are described in Table 3. When the water bonds to $\mathrm{Mn} 1$ (structure 1), the $\mathrm{OO}-\mathrm{H}$ bond length is $1.077 \AA$ indicating that the outside oxygen is of type $\mathrm{O} 1$ and its hydrogen bond to the water is quite weak with the OO-HW separation $2.16 \AA$. However, when the water bonds to $\mathrm{Mn} 2$ (structure 2), the OO-H distance increases to $1.294 \AA$, indicating that the outside oxygen is now of type $\mathrm{O} 2$ so that 
the OO-HW separation decreases to $1.86 \AA$, forming a much stronger hydrogen bond. In both cases the water to oxygen interaction constitutes orthodox hydrogen bonding with $\mathrm{OO}-$ OW separations in excess of $2.8 \AA$; some examples of these interactions are marked on Figure 5 using green lines. The energy decomposition provided in Table 3 indicates that the enhanced binding to the Mn2 site arises from the much stronger interaction of the water and substrate, -16.6 versus $-14.1 \mathrm{kcal}$ $\mathrm{mol}^{-1}$, with the water being slightly more distorted but the bulk surface slightly less distorted. The reduced manganite distortion energy of $4.9 \mathrm{kcal} \mathrm{mol}^{-1}$ for the Mn2 binding compared to 5.5 kcal $\mathrm{mol}^{-1}$ for Mn1 binding is not expected based on the observed proton shift but arises due to structural changes involving the interactions of the second layer with the manganese. Hence the differing chemical properties of the surface manganese atoms significantly influence the surface chemistry.

At high coverage, the water molecules can form a highsymmetry monolayer in which each surface manganese atom is coordinated to a water oxygen, with one water hydrogen coordinating to the surface oxygen $\mathrm{OO}$ while the other hydrogen bonds to another water molecule. A low-energy structure of this type found using PW91 (structure 4) is shown in Figure 5, while each water molecule interacts twice as a Lewis acid and twice as a Lewis base, as is optimal for water interactions, the interaction topology is far from the desired tetrahedral network. A similar structure of just $0.1 \mathrm{kcal} \mathrm{mol}^{-1}$ lower energy is also found (structure $\mathbf{3}$ ), differing from $\mathbf{4}$ only in the relative importance of binding to $\mathrm{Mn} 1$ and $\mathrm{Mn} 2$ sites. While various previous results have indicated that $\mathrm{Mn} 2$ atoms are more strongly bound to the subsurface layer and at low coverage Mn1 atoms are more susceptible to surface reactions, the distinction between the reactivity of $\mathrm{Mn} 1$ and $\mathrm{Mn} 2$ atoms at high coverage is clearly lost. The average interaction energy of surface and water molecules for structure $\mathbf{3}$ is $-11.9 \mathrm{kcal} \mathrm{mol}^{-1}$ of adsorbate, only $0.8 \mathrm{kcal} \mathrm{mol}^{-1}$ more stable than that for water adsorption at low coverage, despite the presence of additional water-water hydrogen bonds. This provides clear evidence that the bonds are strained and hence, in a system of this complexity, alternate structures may be viable. One possible alternate high-symmetry structure (structure 5) is also reported in Table 3: this has the water molecules aligned in planes that approach being perpendicular to the surface (as opposed to the near parallel planes visible in Figure 3). In this structure, each water molecule interacts with the surface via just a single $\mathrm{HW}-\mathrm{OO}$ hydrogen bond while all of the $\mathrm{Mn}-\mathrm{OW}$ bonds are broken. The energy of this configuration was much higher at $-6.1 \mathrm{kcal} \mathrm{mol}^{-1}$, but of this $-4.7 \mathrm{kcal} \mathrm{mol}^{-1}$ arises from the weakening of the waterwater interactions. At high coverage it is not the relative reactivity of the surface atoms but rather the competition between water-surface and water-water interactions that is the most important feature.

Structure 4 shown in Figure 5 has an antiferroelectric arrangement of the surface $\mathrm{OH}$ groups, like the arrangement found in the bulk but unlike that of the raw cleaved surface. However, the higher-energy perpendicularly bonded structure 5 remains similar to that of the raw surface. To investigate this effect, a search was performed for a ferroelectric structure that is directly analogous to $\mathbf{4}$, and properties of the resulting structure, structure $\mathbf{6}$, are shown in Table 3 . While this structure is a true local minimum on the potential energy surface, both PW91 and PM6 tend to optimize configurations in its near vicinity to structure 4 instead. Both structures have similar energy as, from Table 3 , the energy cost associated with making the surface antiferroelectric is opposed by an enhanced water-

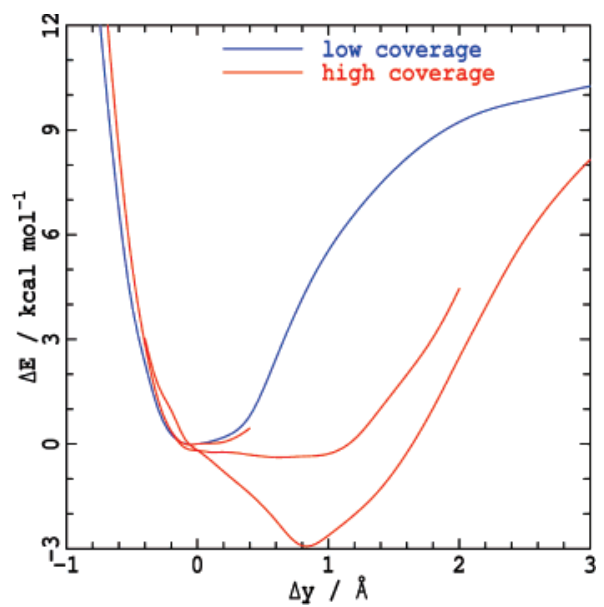

Figure 6. Potential-energy surfaces evaluated using PW91 (soft pseudopotential) for water interacting with the 4-layer manganite (010) surface at either low coverage (starting at structure 1) or high coverage (starting at structure 4). These are obtained by constraining the $y$ (that is, (010)) coordinate of one oxygen atom to be $\Delta y$ from its optimized value while optimizing all other surface and water coordinates. Three surfaces are traced at high coverage for the three different water topologies encountered.

surface interaction. In Figure 3 is the potential-energy surface obtained by linear interpolation between the ferroelectric and antiferroelectric structures is compared to similarly obtained surfaces for bulk hydrogens and for hydrogens on the bare surface. Hydration of the surface reverses the restructuring, making it more bulk-like.

Table 3 also shows results obtained using the hard pseudopotential analogous to structures $\mathbf{4}$ and $\mathbf{6}$, named $\mathbf{4 a}$ and $\mathbf{6 a}$, respectively. As is expected based on the extremely high degree of flexibility of the monolayer structure already found, the choice of pseudopotential is significant. The weaker Mn2-OW bonds break producing a corrugated water layer, but the binding energies of water to the surface predicted by the hard pseudopotential is ca. $-10.3 \mathrm{kcal} \mathrm{mol}^{-1}$, very similar to that predicted by the soft pseudopotential of ca. $-11.8 \mathrm{kcal} \mathrm{mol}^{-1}$.

Figure 6 investigates the process by which water molecules can be removed from the surface under conditions of either low or high coverage. It is constructed starting with structures $\mathbf{1}$ or 4, displacing in the (010) direction the oxygen atom of one water molecule (bonded to Mn2 in each case). This nuclear coordinate is then frozen while all other surface and adsorbate coordinates are reoptimized. The energy profile obtained at low coverage is a simple curve depicting the coordination and hydrogenbonding interactions with the surface. The potential energy changes very slowly with distance near equilibrium, however, as the initial outward displacement weakens the $\mathrm{Mn}-\mathrm{OW}$ coordination but strengthens the $\mathrm{OO}-\mathrm{HW}$ hydrogen bonding. During this process the orientation of the water molecule changes to become more perpendicular with the surface. At high coverage, very complex behavior is found, however, with the hydrogen bonding undergoing subtle rearrangements to produce two additional topologies that support alternate local minima on the potential-energy surface. In fact, Figure 6 shows details of the potential-energy surfaces of each of these topologies. Most significantly, the two newly found topologies show either a very flat potential-energy surface extending up to $1 \AA$ of water displacement or a new deep minimum appearing at $0.8 \AA$ displacement. In both structures, the separated water molecule sits above the plane of the other waters, with its $\mathrm{Mn}-\mathrm{OO}$ link obviously broken (bond length $3.5 \AA$ ), a strained HW-OO hydrogen bond (bond length $2.5 \AA$ ), and strong, properly 


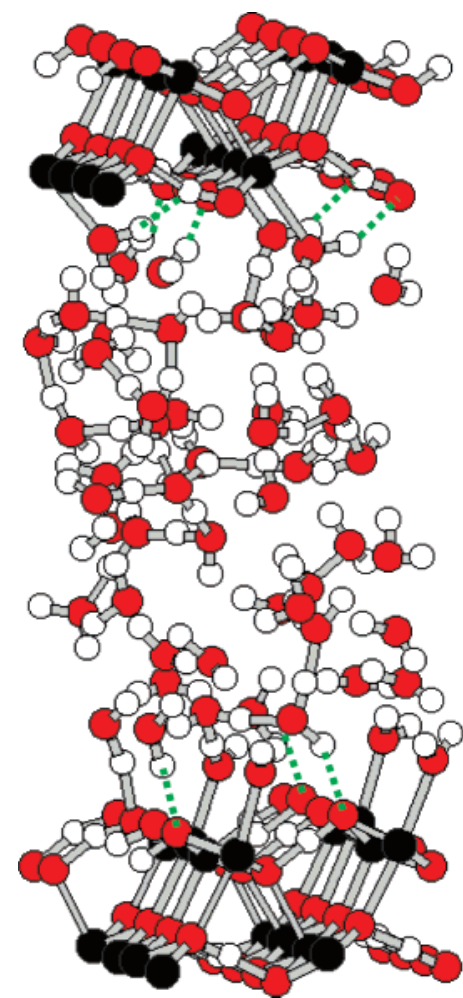

Figure 7. A snapshot showing just one unit cell $(8.840 \AA \times 25.218$ $\AA \times 10.586 \AA$ ) from the PW91 (soft pseudopotential) liquid simulation at $300 \mathrm{~K}$ of the $1 \times 2$ sample containing 56 water molecules. Short hydrogen bonds $<1.6 \AA$ are shown as full bonds while orthodox hydrogen bonds to the surface (up to $2.2 \AA$ length) are shown by green dots.

oriented water-water hydrogen bonds. This again indicates that the nature of the manganite-water interface is controlled by both water-water and surface water interactions. While the surface appears hydrophilic, the strength of the interactions is too weak to alone determine the surface chemistry. Note that the lowest energy structure found using the hard pseudopotential, $\mathbf{4 a}$, is very reminiscent of this structure, and hence the variations of structure described in Table 3 as a result of changes to the pseudopotential reflect more the multi-configurational nature of the surface than the influence of the pseudopotential.

e. The interface of the Manganite (010) Surface with Liquid Water. DFT-MD simulations were run at $300 \mathrm{~K}$ for three representations of liquid water sandwiched between 4-layer manganite slabs: a $1 \times 1$ surface cell containing 18 water molecules, a $1 \times 1$ surface cell containing 28 water molecules in a thicker layer, and this sample doubled in size using a $1 \times$ 2 surface cell containing 56 water molecules. As the intramolecular and intermolecular hydrogen-bonding energetics have been shown to be insensitive to the choice of soft or hard pseudopotentials, only the computationally efficient soft pseudopotentials are used. A sample configuration from the $1 \times 256$ molecule simulation is shown in Figure 7 from which it is apparent that water molecules are able to form a continuous hydrogen-bonded network that spans the manganite surfaces. In this figure, all short $\mathrm{OH}$ bonds of length less than $1.6 \AA$ are marked as full bonds while orthodox hydrogen bonds between water and the surface are marked with green dashed lines. Distorted structures are apparent for the surface manganese, oxygen, and hydrogen atoms, indicating that at $300 \mathrm{~K}$ the surface undergoes considerable motion.

To quantify the liquid structure, calculated radial distribution functions $g(R)$ and coordination numbers $\mathrm{CO}(R)$ are shown in
Figure 8 while these and other properties are summarized in Table 4. The radial distributions functions shown are those depicting the interactions between the two types of surface manganese atoms (Mn1 and Mn2) and water oxygen (OW), the surface outside oxygens (OO) and water hydrogen (HW), water-water hydrogen bonds, and surface internal proton $(\mathrm{H})$ distributions between the surface oxygens $\mathrm{OO}$ and OI. The properties summary included the average number of manganesewater bonds, $\mathrm{OO}-\mathrm{HW}$ bonds to the surface, water-water hydrogen bonds, as well as the fraction of surface hydrogens in antiferroelectric arrangements and the probability of finding water molecules that are simultaneously bonded to manganese atoms and hydrogen bonded to surface oxygen atoms. Also shown for comparison in both the figure and table are results from the optimized monolayer structure $\mathbf{3}$ as well as results for this structure heated to $300 \mathrm{~K}$.

The results obtained for the water monolayer at $0 \mathrm{~K}$ indicate a tight crystallized adsorbate layer with sharp radial correlations at fixed bond lengths (see Table 3 ) and specific coordination numbers: the sample is entirely antiferroelectric, each surface manganese and $\mathrm{OO}$ atom is coordinated to one water molecule that bridges the two types of coordination sites, and every water molecule donates and receives a hydrogen bond to/from other water molecules. Heating to $300 \mathrm{~K}$ introduces large-scale changes, however, as could be anticipated from the results presented earlier in Table 3 and Figure 6. One-third of the Mn1OW bonds break while three-quarters of the Mn2-OW bonds are broken. Only $60 \%$ of both the OO-HW and OW-HW hydrogen bonds remain, and the surface is antiferroelectric only $40 \%$ of the time. A variety of factors lead to these changes in the structure including the similar energy of the ferroelectric and antiferroelectric surfaces, the thermal energy, and the tendency of the water molecules to form a small number of strong bonds rather then have four weak interactions per molecule.

For the liquid samples considered, these effects are enhanced. Three samples were chosen for study as the volume available to the water molecules is relatively small and hence interference effects can arise from water molecules simultaneously feeling the effects of both surfaces or simultaneously feeling the effects of another water molecule and its periodic image. The smallest sample considered contains just 18 water molecules while the largest contains 56. Surprisingly, the calculated radial distribution functions shown in Figure 8 are very similar for all samples, indicating that even tiny sample sizes yield realistic results. The largest difference found is for the water-water hydrogenbonding $\mathrm{OW}-\mathrm{OW}$ distribution which has an absent first trough at ca. $3 \AA$ in the smallest sample; the other samples contain an enhanced spacing between the manganite surfaces and facilitate a more reasonable water structure. ${ }^{17}$ More quantitatively, the results shown in Table 4 indicate that the smallest sample contains more manganese-water interactions, more manganitewater-manganite cyclic structures, and less water-water hydrogen bonds than do the other samples. For the larger samples, less than half of the manganese-water bonds remain, two-thirds of the $\mathrm{OO}-$ water hydrogen bonds remain, and only $10-20 \%$ of the structures involve water molecules multiply interacting with the surface. However, each water molecule forms on average 1.7 hydrogen bonds to other water molecules, only slightly short of the value of 2 found in ice for tetrahedrally arranged water molecules. In addition, the angular orientations of the water molecules at the surface are qualitatively reminiscent of those found at the liquid-vapor interface. ${ }^{17}$

One limitation of these simulations is the length of time, 1-5 ps, for which the trajectories were propagated. This time is 


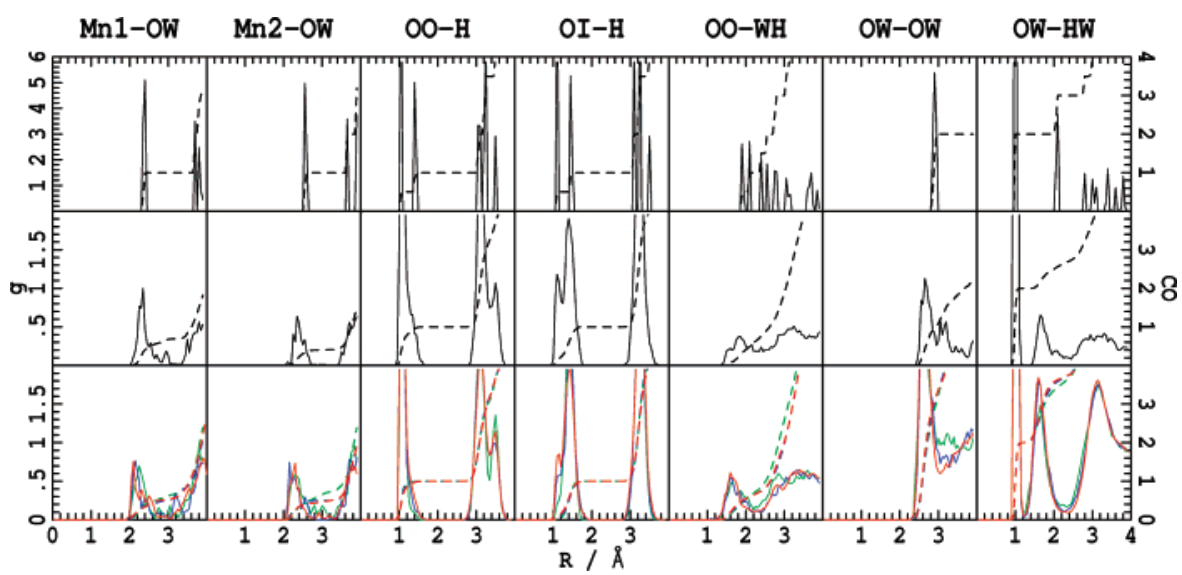

Figure 8. Radial distribution functions $g(R)$ (solid lines) and coordination numbers $\mathrm{CO}(R)$ (dashed lines) calculated using PW91 (soft pseudopotential) for interactions involving surface manganese atoms Mn1 and Mn2, surface oxygen atoms $\mathrm{OO}$ and OI, surface hydrogen atoms $\mathrm{H}$, and water hydrogen and oxygen atoms HW and OW, respectively. Top panel, monolayer structure 3 at $0 \mathrm{~K}$; middle panel, monolayer at $300 \mathrm{~K}$; lower panel, liquid 1 $\times 1$ surface cell containing 18 waters (green), $1 \times 1$ surface cell containing 28 waters (blue), and $1 \times 2$ surface cell containing 56 waters (red).

TABLE 4: Fractions of Occupied Bonds and Bonding Loops Obtained from DFT-MD Simulations at Temperature $T$ of Water Monolayers and Liquid Water Above the Manganite (010) Surface

\begin{tabular}{|c|c|c|c|c|c|c|c|c|c|c|}
\hline sample & $\begin{array}{c}\# \\
\text { waters }^{a}\end{array}$ & $\begin{array}{l}\text { cell } \\
\text { size }^{b}\end{array}$ & $\begin{array}{c}T \\
(\mathrm{~K})\end{array}$ & $\mathrm{aFE}^{c}$ & $\mathrm{Mn} 1-\mathrm{OW}^{d}$ & $\mathrm{Mn} 1-\mathrm{W}-\mathrm{OO}^{e}$ & $\mathrm{Mn} 2-\mathrm{OW}^{d}$ & $\mathrm{Mn} 2-\mathrm{W}-\mathrm{OO}^{e}$ & $\mathrm{OO}-\mathrm{WH}^{d}$ & $\mathrm{OW}-\mathrm{HW}^{f}$ \\
\hline monolayers $^{g}$ & 8 & $1 \times 1$ & 0 & 1.00 & 1.00 & 1.00 & 1.00 & 1.00 & 1.00 & 1.00 \\
\hline monolayers & 8 & $1 \times 1$ & 300 & 0.43 & 0.64 & 0.35 & 0.26 & 0.16 & 0.58 & 0.62 \\
\hline liquid & 18 & $1 \times 1$ & 300 & 0.09 & 0.49 & 0.17 & 0.47 & 0.35 & 0.69 & 1.52 \\
\hline liquid & 28 & $1 \times 1$ & 300 & 0.11 & 0.42 & 0.11 & 0.46 & 0.12 & 0.64 & 1.65 \\
\hline liquid & 56 & $1 \times 2$ & 300 & 0.30 & 0.47 & 0.10 & 0.42 & 0.17 & 0.65 & 1.68 \\
\hline
\end{tabular}

${ }^{a}$ Number of water molecules in the sample. ${ }^{b}$ In terms of the surface-cell component of the orthorhombic bulk-unit cell. ${ }^{c}$ Fraction of configurations with an antiferroelectric arrangement of the surface protons. ${ }^{d}$ Fraction of formed surface-adsorbate interactions involving surface manganese atoms Mn1 and Mn2, surface outside oxygen atoms OO, and water oxygen or hydrogen atoms OW or OH, respectively; 2.6 and $2.2 \AA$ are used for the cutoffs of $\mathrm{Mn}-\mathrm{OW}$ and $\mathrm{OO}-\mathrm{HW}$ hydrogen bonds, respectively. ${ }^{e}$ Fraction of configurations containing doubly coordinated water molecules $\mathrm{Mn}-\mathrm{OW}-\mathrm{HW}-\mathrm{OO} .{ }^{f}$ Average number of water-water hydrogen bonds (using a $2.2 \AA$ cutoff) per water; this is 2 for ice-1h. ${ }^{g}$ Structure 4 from Table 3, as shown in Figure 2.

sufficient to average over gross motions of the water molecules but is insufficient to properly sample all of the possible surfaceliquid interactions. While the qualitative features of the radial distribution functions shown in Figure 8 are robust, the differences indicated in Table 4 between the results for the $1 \times$ 1 sample and its doubled $1 \times 2$ counterpart may be less than the associated uncertainties. A significant aspect of the partially occupied surface coordination sites depicted in Figures 7 and 8 and Table 4 is whether or not any correlation exists involving coordination or lack thereof in one site and the state of its neighboring sites. Unfortunately, these simulations are ca. 2 orders of magnitude too short to be useful in answering such questions, ${ }^{17,34}$ an effect accentuated by the tendency of PW91 to overstructure liquid water, ${ }^{17}$ increasing its relaxation times. ${ }^{34}$ A common motif that rapidly forms upon initial thermalization of structured or completely random samples is one in which all manganese atoms along a (100) vector are either simultaneously bonded or simultaneously nonbonded to water molecules. While the monolayer simulations at $300 \mathrm{~K}$ strongly favor water coordination to $\mathrm{Mn} 1$ atoms rather than to $\mathrm{Mn} 2$ atoms, this effect is not seen in the liquid samples and it is not clear that the apparent result is not attributable to decreased equilibration rates for the monolayer.

\section{Conclusions}

The most characteristic property found for the manganite (010) surface is the weak interactions that it forms with its environment, both interactions within the bulk crystal (the average cross-layer bond energy being just $2.7 \mathrm{kcal} \mathrm{mol}^{-1}$ ) and with liquid water (water preferring to interact with itself rather than the surface). While the weak interlayer forces are somewhat expected as the mineral readily cleaves along (010) planes, the ease with which the surface undergoes acid/base reactions, its protic nature, and the significant degree of ionicity of the crystal all suggest that manganite should bind strongly to water. If manganite did bind strongly to water, then all processes in aqueous solution involving the formation of surface adsorbates would be influenced by the properties of the displaced water molecules. Instead, we find that water-water interactions control the aqueous interface, and that water takes on liquid-like properties within short distances of the surface. These results suggest that dielectric solvation modes may be quite adequate in describing the properties of adsorbates on the manganite (010) surface; such methods have already been applied ${ }^{8}$ to examine zinc and cadmium adsorption by the surface. ${ }^{4-7}$

However, the local structure of the surface, and hence the local chemical properties, is also shown to be very sensitive to environment. Within each crystal layer the protons take on an antiferroelectric structure in the bulk, but cleavage of the surface results in ferroelectric alignment. Pacification of the surface with water reverses this process, with the calculations predicting almost no energy separation between ferroelectric and antiferroelectric structures upon water coordination. Hence the local water environment is predicted to control the surface structure. This may make it difficult to predict the nature of the active site for reactions in aqueous solution on the surface as a vast range of possible configurations are feasible. Also, it is clear that a wide range of local water coordination structures is feasible at room temperature, and any of these structures may turn out to be critical to some surface chemical process. If this 
is indeed the case, then dielectric continuum models could only be applied to approximate the effects of water molecules beyond the inner coordination sphere. A key test for the accuracy of any dielectric-continuum approach would be the lifting of the surface reconstruction effects associated with the bare surface, and the distinction between the reactivity of bare-surface manganese atoms connected to $\mathrm{O} 1$ or $\mathrm{O} 2$ atoms in the subsurface layer.

Two considerably different computational methods were used in these calculations, the PW91 DFT method which is a very commonly used approach for modeling bulk metallic, ionic, and molecular solids and their surfaces, and a beta-release version of PM6, an efficient but more approximate scheme that makes, for the first time, transition metal such as manganese amenable at this level of calculation. Both methods provide qualitatively similar results, indicating weak manganite interlayer binding and weak surface-water interactions, but while PW91 predicts a positive interlayer bond strengths of $2.7 \mathrm{kcal} \mathrm{mol}^{-1}$, PM6 fails to properly bind the (010) layers making this method difficult to apply to manganite interactions. It is likely that small changes to the PM6 parametrization of manganese could result in significantly enhanced calculated properties for manganite and perhaps also other manganese minerals, and this semiempirical approach does appear to have promise as a quick but general a priori computational method for use in materials science. Certainly, the most significant drawback from the DFT calculations is the computer time required for the MD, especially if use of the hard oxygen pseudopotential is essential. The primary accuracy limitations of the PW91 functional relate to its overstructuring of liquid water ${ }^{17}$ and, despite its good performance for hydrogen-bond energies, its tendency to shorten intermolecular $\mathrm{O}-\mathrm{O}$ hydrogen-bonding separations. ${ }^{26-31}$ These properties may be improved through use of more modern density functionals, ${ }^{29,34}$ when they become more widely available. If pseudopotentials are used in conjunction with PW91, it is clear that only hard oxygen pseudopotentials can correctly represent $\mathrm{OH}$ covalent and hydrogen-bonding bond lengths, vibration frequencies, and transition states, but soft pseudopotentials appear adequate for the relative energetics of local minima. Nevertheless, PW91 appears to overestimate the hydrogen-bond strengths in this system. Proper treatment of anharmonicity is critical not only for the description of $\mathrm{OH}$ vibrations in molecules and complexes but is also critical to the understanding of band positions and band widths in water clusters and especially in liquid water, ${ }^{35,36}$ so traditional methods such as PW91 still remain useful for detailed analyses of experimental spectroscopic data.

Acknowledgment. We thank the Australian Research Council and National Natural Science Foundation of China (Grant No.: 20677053) for funding this research and the Australian Partnership for Advanced Computing (APAC) and Australian Centre for Advanced Computing and Communications (AC3) for the provision of computational resources. We thank Dr. J. J. P. Stewart for providing the beta release of MOPAC 7.2 and for modifying the program to allow for external manipulation of the initial density matrices.

Supporting Information Available: The coordinates optimized using PW91 for the bulk solid, the hydrated surfaces, and representative configurations for the sandwiched water liquids provided as ASCII text files. This material is available free of charge via the Internet at http://pubs.acs.org.

\section{References and Notes}

(1) Menard, H. W.; Shipek, C. J. Nature 1958, 182, 1156.

(2) Post, J. E. Proc. Natl. Proc. Sci. U.S.A. 1999, 96, 3447. 166,91

(3) Balachandran, D.; Morgan, D.; Ceder, G. J. Solid State Chem. 2002,

(4) Ramstedt, M.; Andersson, B. M.; Shchukarev, A.; Sjoberg, S. Langmuir 2004, 20, 8224.

(5) Pan, G.; Qin, Y.; Li, X.; Hu, T.; Wu, Z.; Xie, Y. J. Colloid Interface Sci. 2004, 271, 28 .

(6) Bochatay, L.; Persson, P.; Sjoberg, S. J. Colloid Interface Sci. 2000, 229, 584.

(7) Bochatay, L.; Persson, P. J. Colloid Interface Sci. 2000, 229, 593.

(8) Zhu, M.-Q.; Pan, G.; Liu, T.; Li, X.-L.; Yang, Y.-H.; Li, W.; Li, J.; Hu, T.-D.; Wu, Z.-Y.; Xie, Y.-N. Acta Phys.-Chim. Sin. 2005, 21 1378.

(9) Li, X.; Pan, G.; Qin, Y.; Hu, T.; Wu, Z.; Xie, Y. J. Colloid Interface Sci. 2004, 271, 35.

(10) Berry, L. G.; Mason, B.; Diethich, R. V. In Mineralogy-Concepts, Descriptions, Determinations, 2nd ed.; Freeman and Co.: San Francisco, CA, 1983.

(11) Dachs, H. Z. Kristallogr. 1963, 118, 303.

(12) Kohler, T.; Armbruster, T.; Libowitzky, E. J. Solid State Chem. 1997, 133, 486.

(13) Jun, Y.-S.; Martin, S. T. Environ. Sci. Technol. 2003, 37, 2363.

(14) Perdew, J. P.; Wang, Y. Phys. Rev. B 1992, 45, 13244.

(15) Libowitzky, E. Monatsh. Chem. 1999, 130, 1047.

(16) Libowitzky, E.; Rossman, G. R. Amer. Mineral. 1997, 82, 1111.

(17) Vassilev, P.; Hartnig, C.; Koper, M. T. M.; Frechard, F.; van Santen, R. A. J. Chem. Phys. 2001, 115, 9815.

(18) Reimers, J. R.; Cai, Z.-L.; Bilic, A.; Hush, N. S. Ann. N.Y. Acad. Sci. 2003, 1006, 235.

(19) Stewart, J. J. P. MOPAC 7.2 Beta Version; Stewart Computational Chemistry: Colorado Springs, 2006.

(20) Kresse, G.; Hafner, J. Phys. Rev. B 1993, 47, RC558.

(21) Kresse, G.; Furthmüller, J. Comput. Mater. Sci. 1996, 6, 15.

(22) Vanderbilt, D. Phys. Rev. B 1990, 41, 7892.

(23) Kresse, G.; Hafner, J. J. Phys. Condens.: Matter 1994, 6, 8245.

(24) HYPERCHEM 5.1 Pro for Windows; Hypercube Inc.: Gainesville, FL, 1997.

(25) Cai, Z.-L.; Reimers, J. R. J. Chem. Phys. 2000, 112, 527.

(26) van der Wijst, T.; Guerra, C. F.; Swart, M.; Bickelhaupt, F. M. Chem. Phys. Lett. 2006, 426, 415.

(27) Crittenden, D. L.; Thompson, K. C.; Jordan, M. J. T. J. Phys. Chem A 2005, 109, 2971.

(28) Singer, S. J.; Kuo, J.-L.; Hirsch, T. K.; Knight, C.; Ojamaee, L.; Klein, M. L. Phys. Rev. Lett. 2005, 94, 135701/1.

(29) Zhao, Y.; Tishchenko, O.; Truhlar, D. G. J. Phys. Chem. B 2005 , 109, 19046.

(30) Tsuzuki, S.; Luthi, H. P. J. Chem. Phys. 2001, 114, 3949.

(31) Johnson, E. R.; DiLabio, G. A. Chem. Phys. Lett. 2006, 419, 333.

(32) Henkelman, G.; Arnaldsson, A.; Jonsson, H. Comp. Mater. Sci. 2006, 36, 354.

(33) Bader, R. Atoms in Molecules: A Quantum Theory; Oxford University Press: New York, 1990.

(34) Vondele, J. V.; Mohamed, F.; Krack, M.; Hutter, J.; Sprik, M.; Parrinello, M. J. Chem. Phys. 2005, 122, 014515.

(35) Reimers, J. R.; Watts, R. O. Chem. Phys. 1984, 91, 201.

(36) Zhu, M.; Pan, G. J. Phys. Chem. A 2005, 33, 7648. 